\title{
Group B streptococcal bacteraemia in the elderly
}

\author{
C. TRIVAlle, E. MARTIN*, P. MARTEL*, B. JACQUE*, J. F. MENARD† and J. F. LEMELAND \\ Service de Médecine Gériatrique, * Laboratoire de Bactériologie, †Département de Statistique, Centre \\ Hospitalier et Universitaire de Rouen, 76031 Rouen Cedex, France
}

\begin{abstract}
The aim of this retrospective study was to determine the clinical spectrum of group B streptococcal (GBS) bacteraemia in patients over 70 years old. Sixty-six adults with GBS bacteraemia were reviewed over a 5-year period. Disease characteristics, clinical diagnoses and underlying disease were compared in 33 older patients (mean age 82.4 years) and 33 younger patients (mean age 54.2 years). The older patients were also compared with a control group (mean age 81.3 years). Urinary tract infection (39\%), skin infection (33\%) and pneumonia (24\%) were the most frequent clinical diagnoses in older patients. Urinary tract infection (39\% versus $6 \%$ ) was significantly more frequent in older than in younger patients. One underlying disease and one condition were more frequent in elderly patients: congestive heart failure $(39 \%$ versus $6 \%)$ and being bedridden (36\% versus $0 \%$ ). A comparison with the older control group showed that being bedridden was highly associated with GBS bacteraemia and was an important mortality factor amongst older patients $(10 \%$ versus $30 \%)$. In conclusion, GBS disease in the elderly was found to be a severe clinical problem with a high mortality despite appropriate treatment.
\end{abstract}

\section{Introduction}

The Lancefield group B streptococcus (GBS, Streptococcus agalactiae) is a well-documented cause of neonatal and postpartum infections. GBS infections in adults have also been reported increasingly [1-4]. The clinical spectrum includes skin and soft-tissue infection, urinary tract infection, pneumonia, meningitis, septic arthritis, endocarditis and bacteraemia of unknown origin. Specific defects in host immunity caused by diabetes mellitus, cancer or liver disease may predispose certain adults to GBS infection.

In a recent study in the USA, the annual incidence of invasive GBS was estimated at 4.4/100000 adults and $18 / 100000$ in patients $\geqslant 60$ years old [2]. This incidence approaches that of bacteraemia caused by $S$. pneumoniae in American adults.

Received 20 Oct. 1997; accepted 27 Nov. 1997.

Corresponding author: $\mathrm{Dr}$ C. Trivalle. Present address: Service de Gérontologie du Dr Sebag-Lanoë, Hôpital Paul Brousse, 14 Av. Paul Vaillant-Couturier, 94804 Villejuif Cedex, France.

Presented at the 15th Interdisciplinary Meeting on AntiInfectious Chemotherapy, Paris, France, December 1995.
The aim of this retrospective study was to determine the clinical spectrum of GBS bacteraemia in patients $\geqslant 70$ years old.

\section{Patients and methods}

All non-pregnant adults ( $>15$ years old) admitted to the University Hospital of Rouen, for whom blood cultures were positive for GBS during the period July 1990-July 1995, were studied. The University Hospital of Rouen is a 2362-bed tertiary care hospital in the west of France. The population of the Rouen area at that time was $c .440,000$.

The clinical chart of each patient who had documented bacteraemia during the study period was reviewed. Clinical diagnosis, information on underlying disease and outcome were determined from the physician's notes or the discharge summary. A bedridden state was defined by a Karnofsky score of 10-30. Each patient was counted once, even if GBS was isolated from multiple sites or on multiple occasions. Bacteraemia that occurred $>48 \mathrm{~h}$ after admission was defined as nosocomial. GBS were isolated and identified by routine techniques. Antibiotic susceptibility testing for GBS was performed by the KirbyBauer disk-diffusion method. 
The study population was subdivided into two groups: patients $\leqslant 70$ years old and patients $>70$ years old. Both groups were compared for sex ratio, type of infection, underlying disease, antibiotic susceptibilities and prognosis. The older patients were also compared with 99 control patients to determine specific risk factors. These controls were older GBS-negative patients (mean age: 81.3 SD 5.2 years) matched to the older cases on a 3:1 basis by sex, age \pm 2 years, date of admission \pm 2 days and admission to the same general service as the study patient.

Data were analysed by $\chi^{2}$ or Fisher's exact test when samples were not sufficiently large, and variables were expressed as the mean and SD and were compared by Student's $t$ test. A p value of $<0.05$ was considered statistically significant. For the comparison of diagnosis $(n=11)$ and underlying diseases $(n=12)$ (Table 1 ), a $\mathrm{p}$ value $<0.004$ was considered statistically significant after Bonferroni correction for multiple comparisons. Odds ratio (OR) and $95 \%$ confidence intervals (CI) were calculated by the Miettinen method.

\section{Results}

During the 5-year study period, there were 194 cases of GBS bacteraemia in the hospital, accounting for $1.7 \%$ of all cases of bacteraemia. Overall, $66(34 \%)$ of the cases occurred among adults. The annual incidence of GBS bacteraemia in patients $\geqslant 60$ years old in the Rouen area ranged from $11.5 / 100000$ during 1991 to 23/100000 during 1994. A comparison between older patients and younger patients is summarised in Table 1. The mean number of underlying diseases for each patient was 1.9 SD 1.6 There was no difference between older (2.1 SD 1.6) and younger patients (1.7 SD 1.6; $\mathrm{p}=0.363$ ).

GBS were isolated from the blood of the 66 study patients. Among these patients, additional sites from

Table 1. Characteristics, clinical diagnoses and underlying diseases in adults with GBS bacteraemia; comparison of younger and older patients

\begin{tabular}{|c|c|c|c|c|}
\hline \multirow[b]{2}{*}{ Variables } & \multicolumn{4}{|c|}{ Number $(\%)$ of patients } \\
\hline & $\begin{array}{c}\text { All } \\
(n=66)\end{array}$ & $\begin{array}{c}>70 \text { years old } \\
(n=33)\end{array}$ & $\begin{array}{c}\leqslant 70 \text { years old } \\
(n=33)\end{array}$ & $\mathrm{p}$ value* \\
\hline \multicolumn{5}{|l|}{ Characteristic } \\
\hline Mean age (years) & 68.7 SD 17.9 & $82.4 \mathrm{SD} 6.5$ & $54.2 \mathrm{SD} 14.0$ & NA \\
\hline Sex (female) & $32(48)$ & $21(64)$ & $11(33)$ & 0.027 \\
\hline Fever $^{\dagger}$ & $64(97)$ & $32(97)$ & $32(97)$ & 1 \\
\hline Septic shock & $3(4.5)$ & $2(6)$ & $1(3)$ & 1 \\
\hline Nosocomial infection & $20(30.3)$ & $8(24.3)$ & $12(36.4)$ & 0.422 \\
\hline Polymicrobial bacteraemia & $4(6)$ & $2(6)$ & $2(6)$ & 1 \\
\hline Recurrent infection & $2(3)$ & $2(6)$ & $0(0)$ & 0.473 \\
\hline Deaths & $18(27.3)$ & $10(30.3)$ & $8(24.3)$ & 0.782 \\
\hline \multicolumn{5}{|l|}{ Diagnosis $^{\ddagger}$} \\
\hline Skin infection & $19(28.9)$ & $11(33.3)$ & $8(24.2)$ & 0.587 \\
\hline Urinary tract infection & $15(22.7)$ & $13(39.4)$ & $2(6.1)$ & 0.003 \\
\hline No identified source & $9(13.6)$ & $1(3)$ & $8(24.2)$ & 0.031 \\
\hline Pneumonia & $9(13.6)$ & $8(24.2)$ & $1(3)$ & 0.031 \\
\hline Arthritis & $8(12.1)$ & $2(6.1)$ & $6(18.2)$ & 0.258 \\
\hline Surgery-related & $5(7.5)$ & $2(6.1)$ & $3(9.1)$ & 1 \\
\hline ENT infection ${ }^{\S}$ & $3(4.5)$ & $0(0)$ & $3(9.1)$ & 0.237 \\
\hline Meningitis & $3(4.5)$ & $3(9.1)$ & $0(0)$ & 0.237 \\
\hline Endocarditis & $3(3.4)$ & $1(3)$ & $2(6.1)$ & 1 \\
\hline Intravenous catheter infection & $2(3)$ & $1(3)$ & $1(3)$ & 1 \\
\hline Peritonitis & $1(1.5)$ & $0(0)$ & $1(3)$ & 1 \\
\hline \multicolumn{5}{|l|}{ Underlying diseases } \\
\hline Neoplastic disease & $17(25.8)$ & $6(18.2)$ & $11(33.3)$ & 0.26 \\
\hline Congestive heart failure & $15(22.7)$ & $13(39.4)$ & $2(6.1)$ & 0.003 \\
\hline Diabetes mellitus & $15(22.7)$ & $6(18.2)$ & $9(27.3)$ & 0.514 \\
\hline Bedridden state & $12(18.2)$ & $12(36.4)$ & $0(0)$ & 0.0001 \\
\hline Alcohol abuse & $12(18.2)$ & $0(0)$ & $12(36.4)$ & 0.0001 \\
\hline Skin disease & $10(15.1)$ & $6(18.2)$ & $4(12.1)$ & 0.771 \\
\hline Neurological disease & $9(13.6)$ & $5(15.1)$ & $4(12.1)$ & 1 \\
\hline Liver disease & $7(10.6)$ & $0(0)$ & $7(21.2)$ & 0.016 \\
\hline Dementia & $6(9.1)$ & $5(15.1)$ & $1(3)$ & 0.199 \\
\hline Urinary incontinence & $6(9.1)$ & $5(15.1)$ & $1(3)$ & 0.199 \\
\hline Pulmonary disease & $3(4.5)$ & $1(3)$ & $2(6.1)$ & 1 \\
\hline Chronic renal failure & $1(1.5)$ & $0(0)$ & $1(3)$ & 1 \\
\hline
\end{tabular}

NA, not accurate.

*After Bonferroni correction for multiple comparisons, a $\mathrm{p}$ value $<0.004$ was considered statistically significant for the comparison of diagnosis $(n=11)$ and underlying diseases $(n=12)$.

${ }^{\dagger}$ Two patients had hypothermia.

${ }^{\ddagger}$ Patient may have more than one diagnosis.

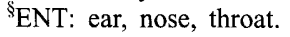


which GBS had grown included urine (12 patients), soft tissue or bone (four), cerebrospinal fluid (two), synovial fluid (two), peritoneal fluid (one) and sputum (one).

The main underlying diseases for the 99 older controls were: congestive heart failure $(\mathrm{n}=31)$, dementia $(n=22)$, neoplastic disease $(n=16)$, chronic renal failure $(\mathrm{n}=13)$, bedridden state $(\mathrm{n}=11)$, neurological disease $(n=11)$, urinary incontinence $(n=11)$ and diabetes mellitus $(n=8)$. The comparison between older patients and older controls showed only one underlying condition which was highly associated with GBS bacteraemia: a bedridden state (OR 4.57; 95\% CI $1.77-11.78 ; p<0.002)$. There was no difference in the incidence of neoplastic disease or diabetes mellitus in the two groups.

All the patients with GBS bacteraemia were treated intravenously with penicillin $G$ and aminoglycosides (other than streptomycin) for 15 days. The course of antibiotic therapy was extended for endocarditis. During hospitalisation, the mortality rate was $27 \%$, with no significant difference between older and younger patients (Table 1). However, the mortality rate was significantly higher for the older patients with GBS bacteraemia than for older controls $(30.3 \%$ versus $10.1 \% ; \mathrm{p}<0.012)$. During the study, two of 23 elderly survivors $(8.7 \%)$ had recurrent GBS bacteraemia.

Of the 66 isolates tested, all were susceptible to penicillin $\mathrm{G}$ and ampicillin ( $\mathrm{MIC}<0.1 \mathrm{mg} / \mathrm{L}$ ). Thirteen isolates $(20 \%)$ showed a high level of resistance to at least one aminoglycoside (more frequently streptomycin). There was no significant difference amongst isolates from the two age groups.

\section{Discussion}

This adult population with GBS bacteraemia was comparable with previous documented studies [1-3]. The male predominance amongst young patients is perhaps due to the exclusion of pregnant females. The female predominance amongst patients $>70$ years old corresponds with the sex ratio of the general elderly population in France. The frequency of nosocomial infection in the present study $(30 \%)$ is between the interval of 17 and $70 \%$ reported in the literature [4]. Therefore, GBS should be considered a potential cause of nosocomial infections in elderly patients $(24 \%)$ as well as in young patients $(36 \%)$.

Urinary tract infection, skin infection (including cellulitis, foot ulcers and pressure ulcers) and pneumonia were the most frequently reported diseases associated with elderly GBS bacteraemia in this study. These observations support the importance of genitourinary and skin colonisation by the organism in males and females. GBS pneumonia generally occurs in older adults with neurological illness or dementia. This suggests that aspiration is an important pathogenic mechanism in GBS pneumonia [2,4]. GBS meningitis remains uncommon in older patients. Bacteraemia with an unidentified source is a common clinical presentation in adults [1-3], but was rare amongst these elderly patients.

The present study identified one underlying medical condition (bedridden state) that is significantly more common among older patients with invasive GBS disease than other older hospitalised persons. This condition enhanced the development of pressure ulcers and necrotising soft-tissue infections. In the study by Jackson et al. [3], decubitus ulcers were independently associated with GBS disease (OR 4). A bedridden state is also a risk factor for urinary tract infection and for pneumonia by aspiration. In the study by Verghese et al. [5], all seven elderly patients with GBS pneumonia were bedridden (average age, 73 years). However, infection with other streptococci notably, group A, group C and group G streptococci has also been documented. These organisms are commonly associated with ulcers, cellulitis and necrotising fasciitis in bedridden elderly patients.

Liver disease and alcohol abuse have been reported as predisposing factors for GBS bacteraemia in adults $[3,4]$. In the present study, these factors were only present in younger patients. The study did not identify any significant association between GBS disease and diabetes mellitus or carcinoma in the elderly group. In previous reports, the presence of diabetes mellitus has been associated with a significantly increased risk of invasive GBS infection in adults of all ages, including the elderly [1-3]. However, it is probable that the magnitude of risk is highest for diabetic patients younger than 45 years of age $[2,3]$.

Age is documented to be an independent risk factor for GBS infection, and persons $\geqslant 65$ years have a significantly higher nosocomial mortality rate than younger persons with GBS infection [3]. However, it is unclear whether mortality is directly related to the infection or is a result of serious disease. In this study, age was not associated with nosocomial mortality, but the mortality was significantly higher in older patients with GBS bacteraemia than in older controls.

Penicillin remains the treatment of choice for serious streptococcal infections. In this study, GBS isolates were uniformly sensitive to penicillin $G$ and ampicillin. Despite the susceptibility of GBS strains to penicillin, there have been several reports of poor clinical responses to appropriate therapy in adults [6]. These failures could be attributed to penicillin tolerance [6]. Aminoglycosides have little or no activity against GBS when used alone, but synergic activity with penicillin has been demonstrated. This 
activity is absent when strains show high levels of resistance to aminoglycosides. This was the case for $20 \%$ of the strains in the present study.

In conclusion, GBS disease in the elderly was found to be a serious problem during the 5-year study, particularly amongst bedridden patients. The results suggest that efforts should be focused upon disease prevention in this high risk group. Little is known about the serotype distribution or genetic relatedness of GBS isolates from elderly patients with invasive disease, and further investigation is required to determine whether or not adults in this high risk group are candidates for vaccination. We are currently undertaking a multicentre prospective serological and molecular typing study of all GBS isolates from elderly patients.

\section{References}

1. Schwartz B, Schuchat A, Oxtoby MJ, Cochi SL, Hightower A, Broome CV. Invasive group B streptococcal disease in adults. A population-based study in metropolitan Atlanta. JAMA 1991; 266: $1112-1114$.

2. Farley MM, Harvey RC, Stull T et al. A population-based assessment of invasive disease due to group B streptococcus in nonpregnant adults. $N$ Engl $J$ Med 1993; 328: 1807-1811.

3. Jackson LA, Hilsdon R, Farley MM et al. Risk factors for group B streptococcal disease in adults. Ann Intern Med 1995; 123: 415-420.

4. Farley MM. Group B streptococcal infection in older patients. Spectrum of disease and management strategies. Drugs Aging 1995; 6: 293-300.

5. Verghese A, Berk SL, Boelen LJ, Smith JK. Group B streptococcal pneumonia in the elderly. Arch Intern Med 1982; 142: $1642-1645$.

6. Betriu C, Gomez M, Sanchez A, Cruceyra A, Romero J, Picazo JJ. Antibiotic resistance and penicillin tolerance in clinical isolates of group B streptococci. Antimicrob Agents Chemother 1994; 38: 2183-2186. 